\title{
Hybrid Position/Force Control for Biped Robot Stabilization with Integrated Center of Mass Dynamics
}

\author{
Felix Sygulla, Robert Wittmann, Philipp Seiwald, Arne-Christoph Hildebrandt, \\ Daniel Wahrmann and Daniel Rixen
}

\begin{abstract}
Traversing uneven terrain with unexpected changes in ground height still poses a major challenge to walking stabilization of humanoid robots. A common approach to balance a biped in such situations is the control of the ground reaction forces at the feet. However, existing solutions for this direct force control scheme do not allow to integrate changing contact areas. Therefore, we propose an explicit formulation for the contact model in task-space. Furthermore, the dynamics of the center of mass is not considered in existing force control approaches. In this work, we present a method to realize contact forces by accelerating the center of mass within the force controller. We show the validity of our explicit contact model in simulation and real-world experiments with our humanoid robot LOLA. The integration of center of mass dynamics shows great reduction of upper-body inclination angles for a late contact experiment with $5.5 \mathrm{~cm}$ change in ground height. We consider our contact model as a starting point for future integration of sensor-based contact information.
\end{abstract}

\section{INTRODUCTION}

Legged robots theoretically have the ability to navigate in very unstructured environments with highly uneven terrain, where conventional wheeled robots may fail to find a feasible path. In practice, however, the stability of biped robots is still rather limited in the presence of unexpected changes in floor height or other disturbances from the environment. One typical approach for the stabilization of a biped robot is the control of the ground reaction forces at the feet, for which the use of a hybrid position/force control scheme has originally been proposed in [1]. In the context of this method, the work in this paper concentrates on the following research questions:

1) How can we formulate an explicit contact model for a hybrid position/force controller, which allows to easily represent changing contact areas at the feet? Based on sensor information (e.g. contact switches), the actual state of the contacts can be measured. By considering this data in the force control, we expect higher performance of the overall system.

2) Is it possible to improve force tracking performance by considering a reduced dynamics model directly in the force controller? This is based on the fact that ground reaction forces may also be generated by accelerating of the center of mass.

We give an overview on the related work in biped walking stabilization in Sec. III Information on the general structure of our walking controller and the biped LOLA is shown in

Technical University of Munich, Chair of Applied Mechanics, Boltzmannstr. 15, 85748 Garching, Germany felix.sygulla@tum.de

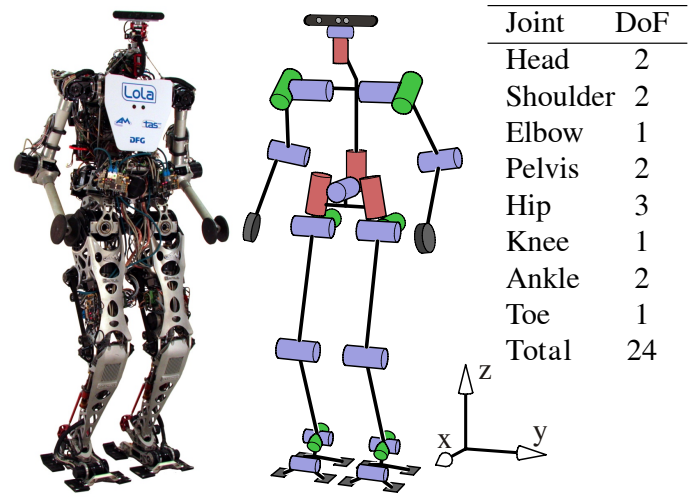

Fig. 1: The humanoid LOLA and its kinematic structure

Sec. III. The proposed explicit contact model and integrated center of mass dynamics are presented in Sec. IV] A simulative and experimental evaluation of our method is conducted in Sec. V] Finally, Sec. VI gives a conclusion to our work.

\section{RELATED WORK}

There exist various publications in the field of biped walking control for the generation of feasible trajectories and the stabilization of the upper body. One prevalent approach is to first generate ideal reference trajectories with reduced models of the biped, which in theory lead to a stable cyclic motion, [2]-[4]. To compensate the deviations caused by the full dynamics of the real robot or external disturbances, feedback of sensory information is utilized. Many approaches for the stabilization of a biped robot have been proposed and experimentally validated on humanoid robots. In the following, we briefly outline the methods we consider most relevant to our contribution.

One common method is to accelerate the center of mass $(\mathrm{CoM})$ to track a reference trajectory for the zero moment point (ZMP) [5]-[7]. However, the effectiveness of this approach is limited by the kinematically feasible region for the CoM. Therefore, other methods adapt the future ZMP reference / step positions in case of large disturbances [8] [10].

Another approach is local torque control at the feet to track reference force trajectories [11]-[14]. This way, the overall contact forces are adapted to match a previously planned motion for the CoM. The ground reaction forces are usually measured by 6-axis force/torque sensors and are controlled by rotating the ankle joints. 
In case of unknown terrain or external disturbances, quick adaptation of the foot position during first ground contact of the swing leg is critical for the stability of a biped. Therefore, several force controllers were designed to specifically reduce the impact during foot landing. This is done directly by modifying the position of the feet [12], [13], [15], [16], or indirectly by modification of joint position control gains during impact [6], [17], [18].

In [12], a stabilization method based on feedback linearization of the multi-body dynamics was proposed. However, the performance of the approach was limited by communication delays and computational power. Thus, an impedance control scheme was used instead to increase the damping of the contacts.

FUJIMOTO ET AL. proposed the use of a hybrid position/force control scheme for the foot torques to track a ZMP reference [1]. Hybrid position/force control was originally formulated for torque-controlled manipulators [19], but the application to position-controlled robots led to the development of hybrid position/force control with inner positionloop, [20]. This control scheme is used in a large number of position-controlled humanoid robots. [13] uses a 2-DOF force controller for the ground reaction torques. In [21], damping control is used on the feet to attain desired ground reaction forces. A similar approach is described in [22], where damping control is used to track reference ZMP trajectories. [14] uses the same balance controller, but extends the force controller to full second-order dynamics.

The direct force control approaches mentioned above do not include a contact model. In contrast, our group's previous publication [23] explicitly describes the contact with the environment by a set of decoupled point contacts of certain stiffness. This has a number of advantages, such as the decoupling of the individual force components and the ability to consider the exact contact geometry. The contact force gradient is described w.r.t. the configuration space $\boldsymbol{q}$, which requires integrated inverse kinematics to attain task-space set points for the inner position loop. Although being an elegant formulation in many ways, the effects of changing contact areas are hard to formalize.

In this work, we introduce a new analytical contact model formulation in task-space for the use in a hybrid position/force control scheme. Additionally, we propose a new method to integrate the center of mass dynamics directly into the force controller.

\section{SYSTEM OVERVIEW}

The method proposed in this paper is experimentally evaluated on the biped LOLA. It weighs approximately $60 \mathrm{~kg}$, is $1.8 \mathrm{~m}$ tall and is actuated by 24 electric joint drives. The kinematic structure of LOLA is shown in Fig. 1] An Inertial Measurement Unit (IMU) is located at the torso of the robot to measure the upper-body state, including inclination $\boldsymbol{\varphi}_{m}$. The robot is equipped with a 6-axis force/torque sensor (FTS) at each foot to measure the ground reaction forces $\boldsymbol{\lambda}_{f, m}$ for each foot $f=\{l, r\}$. For the task-space definition, a torsofixed frame of reference (FoR) $T$ is used. The orientation

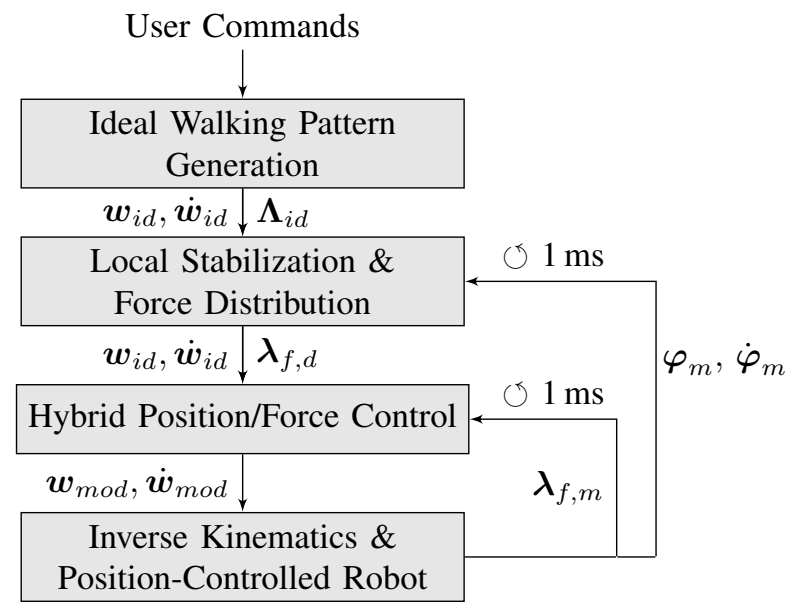

Fig. 2: Overview on the hierarchical walking controller

coincides with the inertial system in Fig. 1, if the upper body is upright. All computations are done on an onboard computer system with the real-time operating system QNX Neutrino. Details on the mechatronic design are described in [24].

The hierarchical control structure is shown in Fig. 2. User inputs given via joystick or step parameters are used to calculate an ideal walking pattern with trajectories $\boldsymbol{w}_{i d}, \dot{\boldsymbol{w}}_{i d}$ for the CoM and the feet as well as the corresponding ideal ground reaction forces $\boldsymbol{\Lambda}_{i d}$. These consist of the ideal force as well as the ideal torques on the CoM $\boldsymbol{\Lambda}_{i d}=\left[\boldsymbol{F}_{i d}, \boldsymbol{T}_{i d}\right]^{T}$. A subsequent local stabilization scheme uses sensor data from the IMU as input and modifies the desired ground reaction forces in order to keep the upper-body upright. Furthermore, the resulting modified forces/torques $\boldsymbol{\Lambda}_{\text {mod }}$ are distributed to the feet using an heuristic approach, [23].

The resulting desired forces/torques $\boldsymbol{\lambda}_{f, d}$ for each foot $f$ are then fed into separate instances of a hybrid position/force control scheme. The included force controller tracks the desired ground reaction forces based on measurements $\boldsymbol{\lambda}_{f, m}$ from the FTS. The force control for each foot modifies the z-position of the foot ${ }_{T} z_{\mathrm{TCP}}$ relative to the center of mass ${ }_{T} z_{\mathrm{CoM}}$, as well as the orientation of the foot $\boldsymbol{w}_{\gamma}$ relative to the torso. To specify the orientation of the foot, the sine values for pitch and tilt angles, $\boldsymbol{w}_{\gamma}=\left[\sin \gamma_{x}, \sin \gamma_{y}\right]$ are used. Thereby, the pitch and tilt angles $\gamma_{y}, \gamma_{x}$ describe independent rotations around the axes of the torso-frame $T$ (for $\gamma_{z} \approx 0$ ). This representation was originally developed by LÖFFLER for the robot Johnnie and is described in [25]. Other components of the task-space representation for the foot, namely the position in $\mathrm{x}$ - and $\mathrm{y}$-direction as well as the rotation around the $\mathrm{z}$-axis remain in the position-controlled space.

The resulting modified task-space trajectories $\boldsymbol{w}_{\text {mod }}, \dot{\boldsymbol{w}}_{\text {mod }}$ are then passed to a velocity-level inverse kinematics based on automatic supervisory control, [26]. Computations on the central control unit run with a sample time of $1 \mathrm{~ms}$. The distributed joint controllers track the trajectories with high local sample rates $(50 \mu$ s for current, 
$100 \mu$ s for velocity and position). For further details on the control system refer to [27].

\section{Extended Hybrid Position/Force Control}

In the following, we first introduce a new explicit contact model formulation in task-space. From a physical point of view, the model is equivalent to the one proposed in [23]. However, it allows straightforward description of changing contact areas for future integration of sensory information. Additionally, we describe a new method to include the CoM dynamics directly in the force control law.

\section{A. Contact Model}

In contrast to most other force control approaches on bipeds, we use an explicit contact model. This allows to easily consider the specific foot geometry \& kinematics, the contact stiffness, as well as the actual state of the contact. Furthermore, the model includes the coupling between the normal force and the torques as the rotation of the foot along one axis does not only influence the respective torque, but can also affect the other force components. For example, if the reference point for rotations is not in the center of a symmetric foot, a rotation will automatically also introduce normal forces on the system. As we assume sufficient friction between ground and foot, the model is formulated specifically for the force-controlled directions, see Sec. III

The contact with the environment is described by a linear elastic stiffness acting on each infinitesimal element $\mathrm{d} A$ of the contact area. This corresponds to an infinite number of decoupled linear springs perpendicular to the ground, which act on the robot's foot, Fig. 3. The contact area of the robot's foot is assumed to be flat, and we choose our "tool center point" (TCP) to reside somewhere on the contact area plane. Within that plane, the position of each contact element is described via $\left.r={ }_{F} x,{ }_{F} y\right]^{T}$ with ${ }_{F} x,{ }_{F} y$ defined in the foot-attached frame $F$. The stiffness $c_{A, z}\left[\mathrm{~N} / \mathrm{m}^{3}\right]$ of these elements can for instance be derived from the elasticity of the contact material $E$ and the effective thickness $d$ of the ground with $c_{A, z}=\frac{E}{d}$. Alternatively, it may be determined experimentally by measuring the vertical stiffness of a ground contact $c_{m, z}$ with a contact area $A: c_{A, z}=\frac{c_{m, z}}{A}$.

For several reasons we do not consider damping effects in our model: (1) The actual damping is hard to estimate. (2) A wrong estimation for the damping may be even worse than not considering damping at all. (3) As the force control acts on velocity-level, disturbances caused by damping effects are reduced effectively (An error in the force results in a changed velocity of the foot).

Consequently, the vertical force on each infinitesimal element $f_{z}$ only depends on the vertical displacement $\Delta z$ of the corresponding contact area $\mathrm{d} A$. The overall deformation of this contact element can be derived as a superposition of the rotational components in vertical direction $\Delta z_{\alpha_{x}}, \Delta z_{\alpha_{y}}$ and the vertical translation $\Delta z_{\mathrm{TCP}}$ of the foot:

$$
f_{z}=c_{A, z} \cdot\left(\Delta z_{\alpha_{x}}+\Delta z_{\alpha_{y}}+\Delta z_{\mathrm{TCP}}\right) .
$$

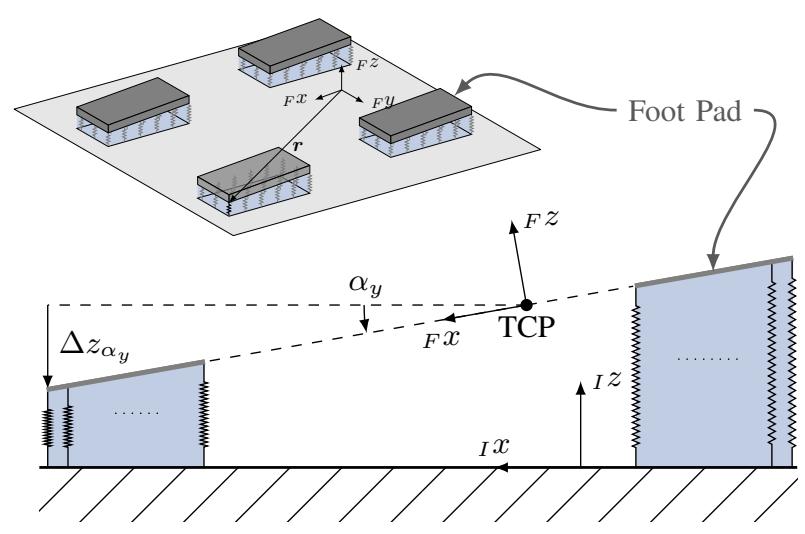

Fig. 3: LoLA's foot with the 4 contact pads and the proposed contact springs in top and side view

Note that superposition of the rotational parts is valid as the angle definition is based on independent roll $\left(\alpha_{x}\right)$ and pitch $\left(\alpha_{y}\right)$ angles in the inertial frame. This matches the definition of the task-space in Sec. III Fig. 3 illustrates the relation between the vertical deformation of one element, the rotation with pitch-angle $\alpha_{y}$ and the translation of the TCP ${ }_{I} z_{\mathrm{TCP}}$ in the inertial frame $I$. Based on geometrical considerations, the vertical force $f_{z}$ can be written as a relation of all three degrees of freedoms, which yields:

$$
f_{z}=c_{A, z} \cdot\left({ }_{F} x \sin \alpha_{y}-{ }_{F} y \sin \alpha_{x}-\left({ }_{I} z_{\mathrm{TCP}}-{ }_{I} z_{0}\right)\right) .
$$

For the normal force caused by translation of the TCP, the difference to an unstressed position ${ }_{I} z_{0}$ is used. Based on the information about $f_{z}$ on each contact area element $\mathrm{d} A=$ $\mathrm{d}_{F} x \mathrm{~d}_{F} y$, the overall torques and force are calculated with a surface integral. This yields three equations for the torques ${ }_{I} T_{x},{ }_{I} T_{y}$ as well as the normal force ${ }_{I} F_{z}$ in the inertial frame:

$$
\begin{aligned}
& { }_{I} T_{x}=\int_{S} f_{z} \cdot{ }_{F} y \cos \alpha_{x} \mathrm{~d} A \\
& { }_{I} T_{y}=-\int_{S} f_{z} \cdot{ }_{F} x \cos \alpha_{y} \mathrm{~d} A \\
& { }_{I} F_{z}=\int_{S} f_{z} \mathrm{~d} A .
\end{aligned}
$$

The cosine parts result from the calculation of the correct lever arm for every $f_{z}$. Inserting (2) into (3) and simplifying yields:

$$
\begin{aligned}
{ }_{I} T_{x} & =c_{A, z} \cos \alpha_{x}[\sin \alpha_{y} \underbrace{\int_{S}{ }_{F} x_{F} y \mathrm{~d} A}_{I_{x y}}-\sin \alpha_{x} \underbrace{\int_{S} F y^{2} \mathrm{~d} A}_{I_{x x}} \\
& -\left({ }_{I} z_{\mathrm{TCP}}-{ }_{I} z_{0}\right) \underbrace{\int_{S} F y \mathrm{~d} A}_{r_{c, y} \cdot A}] .
\end{aligned}
$$

The first two surface integrals can be interpreted as the second moments of area $I_{x y}, I_{x x}$ of the contact area $A$. The third integral contains the ${ }_{F} y$-position $r_{c, y}$ of the center of the contact area with respect to the TCP. Using the same 
relations for the remaining terms ${ }_{I} T_{y}$ and ${ }_{I} F_{z}$ leads to the following linear equation for the contact model of one foot

$$
\boldsymbol{\lambda}_{f}=\left(\begin{array}{c}
{ }_{I} T_{x} \\
{ }_{I} T_{y} \\
{ }_{I} F_{z}
\end{array}\right)=\boldsymbol{U} \cdot\left(\begin{array}{c}
\sin \alpha_{x} \\
\sin \alpha_{y} \\
{ }_{I} z_{\mathrm{TCP}}-{ }_{I} z_{0}
\end{array}\right)=\boldsymbol{U} \cdot \boldsymbol{x}_{f} .
$$

The complete information on the geometry of the foot, material stiffness, the effective contact area (closed contacts) and the coupling between torques and forces is contained in the symmetric matrix $\boldsymbol{U}$. It can be interpreted as the gradient of the torques/forces on the foot $\boldsymbol{\lambda}_{f}$ with respect to inertial movement $\boldsymbol{x}_{f}$ of the foot and generally depends on the cosine of the angles. Within this work, we assume small angles of the feet, which yields

$$
\boldsymbol{U} \approx c_{A, z} \cdot\left(\begin{array}{ccc}
-I_{x x} & I_{x y} & -r_{c, y} A \\
I_{x y} & -I_{y y} & r_{c, x} A \\
-r_{c, y} A & r_{c, x} A & -A
\end{array}\right) .
$$

This gradient can either be considered constant or even be time-variable, e.g. based on contact sensor information. In this paper, we consider $\boldsymbol{U}$ constant by using the foot geometry, i.e. assuming that all parts of the foot have contact with the ground, if the force control is activated. Note that the contact model is only used if the respective foot is on the ground. To retrieve the necessary motions of the foot for a given desired $\boldsymbol{\lambda}_{f}, \boldsymbol{U}$ must be invertible, which is not generally the case. For a time-variable $\boldsymbol{U}$, special measures are necessary to avoid a singular gradient.

In contrast to the definition used here, the original contact model for the force control of LoLA described the gradient of forces/torques with respect to motions in the configuration space, [23]:

$$
\dot{\boldsymbol{\lambda}}=\frac{\partial \boldsymbol{\lambda}}{\partial \boldsymbol{q}} \dot{\boldsymbol{q}}
$$

The gradient is calculated from Jacobians of the contact points and inverse kinematics is required within the hybrid position/force controller in order to retrieve task-space modifications. However, changing contact areas and their effects in task-space are hard to formalize for a gradient in the configuration space $\boldsymbol{q}$. The newly defined model in task-space can be derived from easy to calculate geometric properties of the contact and has an analytical description. We consider this contact model as a starting point for future research on how to integrate dynamically changing contact areas in the force controller.

\section{B. Hybrid Position/Force Control}

The force controller is based on a hybrid position/force control scheme with inner position-control loop in taskspace. To close the force-control loop, the ideal trajectories from the planner module $\boldsymbol{w}_{i d}, \dot{\boldsymbol{w}}_{i d}$ are modified:

$$
\begin{aligned}
& \dot{\boldsymbol{w}}_{\text {mod }}=\dot{\boldsymbol{w}}_{i d}+\Delta \dot{\boldsymbol{w}} \\
& \boldsymbol{w}_{\text {mod }}=\boldsymbol{w}_{i d}+\int \Delta \dot{\boldsymbol{w}} \mathrm{d} t .
\end{aligned}
$$

The controller output $\Delta \dot{\boldsymbol{w}}$ is defined on velocity-level to ensure smooth trajectories. Starting from the full set of workspace components $\boldsymbol{w}$, a binary selection matrix $\boldsymbol{S}_{c, f}$ is used to retrieve the force-controlled directions of a foot $f$ in the workspace

$$
\boldsymbol{w}_{f}=\boldsymbol{S}_{c, f} \boldsymbol{w}=\left(\begin{array}{c}
\sin \gamma_{x} \\
\sin \gamma_{y} \\
T z_{\mathrm{TCP}}-T z_{\mathrm{CoM}}
\end{array}\right)
$$

The corresponding forces/torques $\boldsymbol{\lambda}_{f}$ for the foot can be retrieved from the force/torque sensor readings $\boldsymbol{\lambda}_{f, m}$ with

$$
\boldsymbol{\lambda}_{f}=\boldsymbol{A}_{T F} \boldsymbol{\lambda}_{f, m} .
$$

A projection matrix $\boldsymbol{A}_{T F}$ is needed to transform the forces/torques to the TCP and the torso FoR T. Furthermore, the matrix maps the full 6 -axis force/torque information in $\boldsymbol{\lambda}_{f, m}$ to the force-controlled directions in $\boldsymbol{\lambda}_{f}$. To track the desired forces $\boldsymbol{\lambda}_{f, d}$ on each foot, a first-order linear error dynamics

$$
\delta \dot{e}+e=0, \quad e=\lambda_{f, d}-\lambda_{f}
$$

with time-constant $\delta$ is used. By solving $\sqrt{14}$ for $\dot{\lambda}_{f}$, differentiating as well as inverting the contact-model (7) for constant $\boldsymbol{U}$ and substituting $\dot{\boldsymbol{x}}_{f}=\dot{\boldsymbol{w}}_{f}$, the force control law is given:

$$
\dot{\boldsymbol{w}}_{f}=\boldsymbol{U}^{-1}\left(\dot{\boldsymbol{\lambda}}_{f, d}+\frac{1}{\delta}\left(\boldsymbol{\lambda}_{f, d}-\boldsymbol{\lambda}_{f}\right)\right) .
$$

By mapping the components of $\dot{\boldsymbol{w}}_{f}$ to the components of $\dot{\boldsymbol{x}}_{f}$ in the contact model, we assume small inclination angles of the upper-body as well as ${ }_{T} \dot{z}_{\mathrm{CoM}} \approx 0$. If the walking state of the robot changes from double-support to single-support, the force controller of the swing leg is blended out. Simultaneously, a parallel position-control loop is activated to pull the foot back to the ideally planned trajectory during its swingphase. This results in the following extended control law

$$
\begin{aligned}
\dot{\boldsymbol{w}}_{f, e x t} & =b_{f} \cdot \boldsymbol{U}^{-1}\left(\dot{\boldsymbol{\lambda}}_{f, d}+\frac{1}{\delta}\left(\boldsymbol{\lambda}_{f, d}-\boldsymbol{\lambda}_{f}\right)\right) \\
& +\left(1-b_{f}\right) \cdot\left(\boldsymbol{S}_{c, f} K_{x}\left(\boldsymbol{w}_{i d}-\boldsymbol{w}\right)\right)
\end{aligned}
$$

with the blending factor $b_{f} \in[0,1]$ and the position-control gain $K_{x}$. The blend factor is changed continuously based on the planned contacts of the feet. The trajectory modification for both feet is then given by

$$
\Delta \dot{\boldsymbol{w}}=\sum_{f=\{l, r\}} \boldsymbol{S}_{c, f}^{T} \dot{\boldsymbol{w}}_{f, e x t} .
$$

\section{Integration of CoM Dynamics}

With the explicit contact model and the hybrid position/force control scheme, the ground reaction forces can already be controlled effectively. However, ground reaction forces can also be induced by accelerating the center of mass of the humanoid, which is not considered in the contact model itself. This is of special interest for the single-support phase, where the realizable stabilization torques are limited by the smaller contact surface. We approximate the dynamics of the humanoid in the single-support phase by a single mass $m$ located at the CoM and the moments of inertia ${ }_{T} \theta_{x x}, T_{T} \theta_{y y}$, Fig. 4. We only consider directions which 


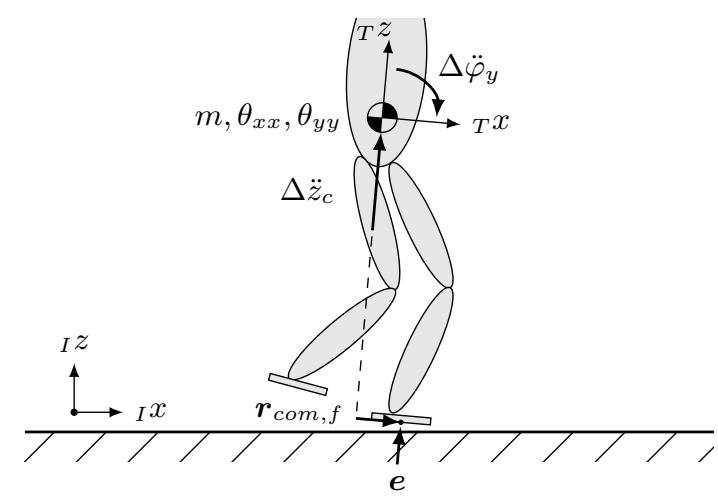

Fig. 4: Reduced model for the CoM dynamics of the humanoid used for integration in the force controller

are actively controlled by the hybrid position/force control. Consequently,

$$
\underbrace{\left(\begin{array}{ccc}
\theta_{x x} & 0 & 0 \\
0 & \theta_{y y} & 0 \\
0 & 0 & m
\end{array}\right)}_{\boldsymbol{M}} \underbrace{\left(\begin{array}{c}
\Delta \ddot{\varphi}_{x} \\
\Delta \ddot{\varphi}_{y} \\
\Delta \ddot{z}_{c}
\end{array}\right)}_{\Delta \boldsymbol{a}}=\underbrace{\left(\begin{array}{ccc}
1 & 0 & T^{T} r_{c o m, f, y} \\
0 & 1 & -T^{r_{c o m, f, x}} \\
0 & 0 & 1
\end{array}\right)}_{\boldsymbol{R}_{f}(t)} \boldsymbol{e}
$$

gives the relation between the error in the contact forces $\boldsymbol{e}$ and the necessary CoM accelerations $\Delta \boldsymbol{a}$ to compensate this error, i.e. the vertical CoM acceleration $\Delta \ddot{z}_{c}$ as well as the rotational accelerations $\Delta \ddot{\varphi}_{x}, \Delta \ddot{\varphi}_{y}$. The vector from the CoM to the stance-foot ${ }_{T}\left[r_{c o m, f, x}, r_{c o m, f, y}\right]^{T}$ in the $x y$ plane and the other quantities are described in the torsofixed frame $T$. By solving (18) for $\Delta \boldsymbol{a}$ and accelerating the stance foot with $-\Delta \boldsymbol{a}$ (relative to the CoM), the desired accelerations of the CoM are attained. Integration over time yields the respective task-space velocities for each foot $f$ :

$$
\Delta \dot{\boldsymbol{w}}_{a, f}=-\int s_{a, f} g_{a} \boldsymbol{M}^{-1} \boldsymbol{R}_{f}(t)\left(\boldsymbol{\lambda}_{f, d}-\boldsymbol{\lambda}_{f}\right) \mathrm{d} t .
$$

The scalar $s_{a, f} \in\{0,1\}$ is a binary activation factor, the gain $g_{a} \in[0,1]$ is used to scale the effects of the method. Equation (19) can be interpreted as an additional integral part to the hybrid position/force controller, where the gain is based on the system's natural CoM dynamics.

The control law can be easily integrated into the existing framework, as the workspace-velocities $\Delta \dot{\boldsymbol{w}}_{a, f}$ are simply added to the output of the direct force control in the hybrid position/force control scheme. However, small foot angles again must be assumed to map the angular velocities in $\Delta \dot{\boldsymbol{w}}_{a, f}$ to the task-space definition $\dot{\boldsymbol{w}}_{\gamma}=$ $\left[\cos \gamma_{x} \dot{\gamma}_{x}, \cos \gamma_{y} \dot{\gamma}_{y}\right]$. The controller is run separately for both feet, with each foot having its own integral state. If $f$ is the stance foot, the corresponding $s_{a, f}$ is set to one, otherwise to zero. Currently, this method is only activated in the singlesupport phase, i.e. both $s_{a, f}$ are set to zero in case both feet are on the ground, or if both are in the air. Hereby, the actual contact state as measured by sensors on the feet is used instead of the planned state. To reset the velocities

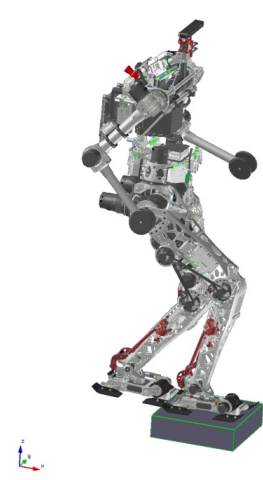

(a) Simulation Scenario



(b) Experimental Scenario
Fig. 5: Test scenarios with a board $(9 \mathrm{~cm}$ height $)$ and a platform $(5.5 \mathrm{~cm}$ height) as disturbances

stored by the integral, the control law is again extended by a parallel position-control loop with gain $K_{a, x}$ :

$$
\begin{aligned}
\Delta \dot{\boldsymbol{w}}_{a, f, e x t} & =-\int s_{a, f} g_{a} \boldsymbol{M}^{-1} \boldsymbol{R}_{f}(t)\left(\boldsymbol{\lambda}_{f, d}-\boldsymbol{\lambda}_{f}\right) \mathrm{d} t \\
& -\int\left(1-s_{a, f}\right) K_{a, x} \Delta \dot{\boldsymbol{w}}_{a, f, e x t} \mathrm{~d} t .
\end{aligned}
$$

It is activated in the double-support phase to quickly remove the remaining velocity modifications on the old stance foot. The overall control law for the hybrid force/torque control with additional integrated CoM dynamics is then given by

$$
\Delta \dot{\boldsymbol{w}}=\sum_{f=\{l, r\}} \boldsymbol{S}_{c, f}^{T}\left(\dot{\boldsymbol{w}}_{f, e x t}+b_{f} \Delta \dot{\boldsymbol{w}}_{a, f, e x t}\right) .
$$

For safety reasons, the modifications $\Delta \dot{\boldsymbol{w}}$ and $\Delta \boldsymbol{w}$ are limited to reasonable values. In case these values are exceeded, an anti-windup logic additionally stops the integration in (20).

\section{RESULTS}

The proposed method was integrated to the control system of the humanoid robot LOLA. In the following, results from our multi-body simulation as well as experimental results are presented. Although a vision system is installed, it was inactive during all tests. For the results shown, only data from IMU, FTS sensors and contact switches on the feet was used on the real-time control unit as well as in simulation. The inertia values in $\boldsymbol{M}$ were calculated based on CADdata. A video of the simulation and conducted experiments is available online: https://youtu.be/SbbVFWSeyLw.

\section{A. Simulation}

For simulation, a full multi-body model of the humanoid robot LOLA with compliant contacts and included jointcontroller dynamics is used. The methods are parametrized with $\delta=0.02 \mathrm{~s}$ and $g_{a}=1$. A phase-reset is activated in case of an early contact with the environment to stop further movement of the foot in direction of the obstacle [16].

First, the validity of the proposed contact model formulation was checked for a walking sequence with unexpected 




Fig. 6: Simulation results without (ref) and with (dyn) integrated CoM dynamics for stepping on an unexpected obstacle of $9 \mathrm{~cm}$ height

unevenness at one step $(0.8 \mathrm{~s}$ step time, $0.4 \mathrm{~m}$ step length, $4 \mathrm{~cm}$ obstacle height) by comparison with the original hybrid position/force implementation [23]. The results show an improvement of root mean square values of the total tracking error for overall torques at the CoM by $\Delta e_{T_{x}} \approx 3 \%$, $\Delta e_{T_{y}} \approx 9 \%$ with the proposed contact model formulation. The inclination angles of the upper-body do not change significantly though.

Based on the controller with new contact model, the influence of the proposed integrated CoM dynamics is analyzed. The simulation scenario is shown in Fig. 5a and includes an unexpected obstacle with $9 \mathrm{~cm}$ height, which is hit at a walking speed of $1.5 \mathrm{~km} / \mathrm{h}$ and a step height of $12 \mathrm{~cm}$. The inclination angles as well as the modifications in the vertical CoM acceleration are depicted in Fig. 6. The obstacle is hit unexpectedly (early contact) at $t_{1}=7 \mathrm{~s}$, which causes the robot to fall backwards with a negative inclination angle. At $t_{2}=7.9 \mathrm{~s}$, the robot stands on the obstacle and expects ground contact with the current swing foot (late contact). This leads to high inclination angles in the reference, as the humanoid tilts along its underactuated DoFs before hitting the actual ground. The integrated CoM dynamics method significantly reduces the inclination of the upper body, both at and directly after the early contact $\left(t_{1}\right)$ as well as in the late contact $\left(t_{2}\right)$ situation. During early contact at $t_{1}$, the forces at the stance foot are too low, as the swing foot unexpectedly touched ground. Therefore, the CoM is accelerated upwards, which in turn also reduces the impact on the swing foot. For the late contact at $t_{2}$, the forces on the stance foot are too high, the CoM is accelerated downwards, which also leads to a faster ground contact of the swing foot. Additionally, the root mean square overall torque tracking errors at the CoM are reduced by $\Delta e_{T_{x}} \approx 21 \%$ and $\Delta e_{T_{y}} \approx 31 \%$.

\section{B. Experiment}

The new contact model formulation and inverse CoM dynamics method were also evaluated on our humanoid robot LOLA. On the real system, we had to reduce the controller gains to $\delta=0.027 \mathrm{~s}, g_{a}=0.8$ to avoid oscillations in still-



Fig. 7: Experimental results without (ref) and with (dyn) integrated CoM dynamics for unexpectedly stepping down a platform of $5.5 \mathrm{~cm}$ height

stand. These are caused by feedback of structural vibrations to the IMU measurements and were also present in the original implementation. As expected, the new contact model formulation showed a behavior equivalent to the contact model in [23] for a normal walking sequence.

The performance of the integrated CoM dynamics was tested in a late contact scenario, Fig. 5b For this, the biped is put on a platform with a height of $5.5 \mathrm{~cm}$ and commanded to walk straight forward with a speed of $1.5 \mathrm{~km} / \mathrm{h}$. The position of the edge of the platform is not detected by any sensors, i.e. the walking pattern generator assumes a flat ground. The phase-reset logic was inactive during the experiments. A comparison of the inclination angle $\varphi_{y}$ for the hybrid position/force control without dynamics (ref) and with dynamics (dyn) is depicted in Fig. 7 The late contact starts at $t \approx 8 \mathrm{~s}$ and results in high inclination angles for the reference controller. With CoM dynamics in the force control scheme, the maximum inclination angles are reduced by $\approx 63 \%$. Furthermore, the disturbance in the inclination angle declines much faster after the late-contact event. Photographs of the experiment are shown in Fig. 8 .

\section{CONCLUSION}

In our contribution, we presented a hybrid position/force control scheme with integrated linear and angular CoM dynamics. This allows the realization of ground reaction forces in the force controller by accelerating the CoM in single-support phase. Simulation results show a significant reduction of the upper-body inclination for early- and late contact situations. In experiments with the biped LOLA, the maximum inclination angle is reduced by $\approx 63 \%$ for a late contact scenario with $5.5 \mathrm{~cm}$ change in ground height.

Additionally, we proposed a new explicit contact model formulation for ground contacts of biped robots. The model is defined in task-space and allows straight-forward consideration of changing contact areas. We validated our model by performance comparison with the explicit contact model proposed in [23]. For the future, we plan to lift the small angles limitations made in this work as well as consider 

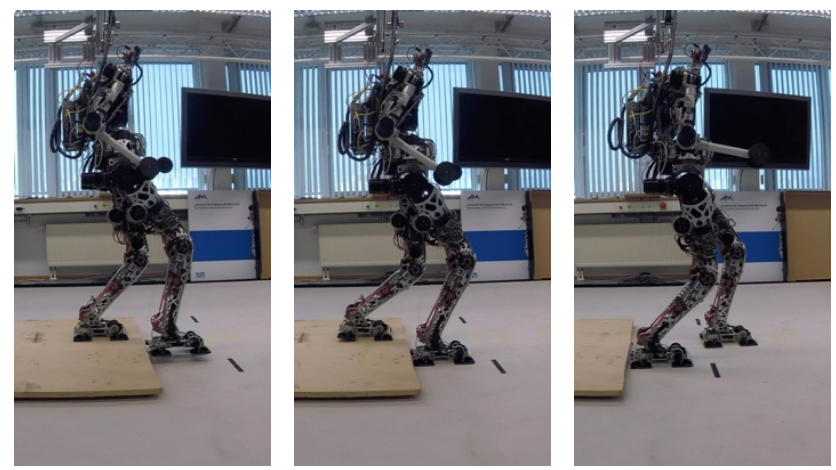

Fig. 8: Photographs of the experiment with activated integrated $\mathrm{CoM}$ dynamics

a time-variable contact gradient $\boldsymbol{U}$ by integration of tactile sensing [28].

\section{REFERENCES}

[1] Y. Fujimoto and A. Kawamura, "Proposal of Biped Walking Control Based on Robust Hybrid Position/Force control," in IEEE International Conference on Robotics and Automation. IEEE, 1996.

[2] S. Kajita, F. Kanehiro, K. Kaneko, K. Fujiwara, K. Harada, K. Yokoi, and H. Hirukawa, "Biped Walking Pattern Generation by using Preview Control of Zero-Moment Point," in IEEE International Conference on Robotics and Automation, 2003.

[3] T. Buschmann, S. Lohmeier, M. Bachmayer, H. Ulbrich, and F. Pfeiffer, "A Collocation Method for Real-Time Walking Pattern Generation," in IEEE-RAS International Conference on Humanoid Robots, 2007.

[4] J. Englsberger and C. Ott, "Integration of vertical COM motion and angular momentum in an extended Capture Point tracking controller for bipedal walking," IEEE-RAS International Conference on Humanoid Robots, 2012.

[5] K. Nishiwaki and S. Kagami, "High Frequency Walking Pattern Generation based on Preview Control of ZMP," in IEEE/RAS International Conference on Humanoid Robots, 2006.

[6] R. Tajima, D. Honda, and K. Suga, "Fast Running Experiments Involving a Humanoid Robot," in IEEE International Conference on Robotics and Automation, 2009.

[7] T. Takenaka, T. Matsumoto, T. Yoshiike, T. Hasegawa, S. Shirokura, H. Kaneko, and A. Orita, "Real Time Motion Generation and Control for Biped Robot -4th report: Integrated Balance Control," in IEEE/RSJ International Conference on Intelligent Robots and Systems, 2009.

[8] K. Nishiwaki and S. Kagami, "Online Walking Control System for Humanoids with Short Cycle Pattern Generation," The International Journal of Robotics Research, 2009.

[9] J. Urata, K. Nishiwaki, Y. Nakanishi, K. Okada, S. Kagami, and M. Inaba, "Online decision of foot placement using singular LQ preview regulation," in IEEE-RAS International Conference on Humanoid Robots, 2011.

[10] R. Wittmann, A.-C. Hildebrandt, D. Wahrmann, D. Rixen, and T. Buschmann, "Real Time Nonlinear Model Predictive Footstep Optimization for Biped Robots," IEEE-RAS International Conference on Humanoid Robots, 2015.
[11] K. Hirai, M. Hirose, Y. Haikawa, and T. Takenaka, "The Development of Honda Humanoid Robot," in IEEE International Conference on Robotics and Automation, 1998.

[12] K. Löffler, M. Gienger, F. Pfeiffer, and H. Ulbrich, "Sensors and Control Concept of a Biped Robot," IEEE Transactions on Industrial Electronics, vol. 51, no. 5, 2004

[13] S. Kajita, T. Nagasaki, K. Kaneko, K. Yokoi, and K. Tanie, "A Running Controller of Humanoid Biped HRP-2LR," in IEEE Internaional Conference on Robotics and Automation, 2005.

[14] M. Morisawa, F. Kanehiro, K. Kaneko, S. Kajita, and K. Yokoi, "Reactive Biped Walking Control for a Collision of a Swinging Foot on Uneven Terrain," in IEEE-RAS International Conference on Humanoid Robots, 2011.

[15] J. Y. Kim, I. W. Park, and J. H. Oh, "Walking Control Algorithm of Biped Humanoid Robot on Uneven and Inclined Floor," Journal of Intelligent and Robotic Systems: Theory and Applications, vol. 48, no. 4, 2007.

[16] T. Buschmann, A. Ewald, H. Ulbrich, and A. Buschges, "Event-Based Walking Control - From Neurobiology to Biped Robots," in IEEE/RSJ International Conference on Intelligent Robots and Systems, 2012.

[17] K. Nishiwaki and S. Kagami, "Sensor Feedback Modification Methods that are Suitable for the Short Cycle Pattern Generation of Humanoid Walking," in IEEE/RSJ International Conference on Intelligent Robots and Systems, 2007.

[18] K. Hashimoto, A. Hayashi, T. Sawato, Y. Yoshimura, T. Asano, K. Hattori, Y. Sugahara, H.-o. Lim, and A. Takanishi, "TerrainAdaptive Control to Reduce Landing Impact Force for HumanCarrying Biped Robot," in IEEE/ASME International Conference on Advanced Intelligent Mechatronics, 2009.

[19] J. J. Craig and M. H. Raibert, "A systematic method of hybrid position/force control of a manipulator," in Computer Software and Applications Conference, 1979. Proceedings. COMPSAC 79. The IEEE Computer Society's Third International, 1979, pp. 446-451.

[20] B. Siciliano, L. Sciavicco, L. Villani, and G. Oriolo, Robotics: Modelling, Planning and Control, ser. Advanced Textbooks in Control and Signal Processing Series. Springer, 2008.

[21] K. Nishiwaki and S. Kagami, "Frequent Walking Pattern Generation that Uses Estimated Actual Posture for Robust Walking Control," in IEEE-RAS International Conference on Humanoid Robots, 2009.

[22] S. Kajita, M. Morisawa, K. Miura, S. Nakaoka, K. Harada, K. Kaneko, F. Kanehiro, and K. Yokoi, "Biped Walking Stabilization Based on Linear Inverted Pendulum Tracking," in IEEE/RSJ International Conference on Intelligent Robots and Systems, vol. 2, 2010.

[23] T. Buschmann, S. Lohmeier, and H. Ulbrich, "Biped Walking Control Based on Hybrid Position/Force Control," IEEE/RSJ International Conference on Intelligent Robots and Systems, 2009.

[24] S. Lohmeier, T. Buschmann, and H. Ulbrich, "System design and control of anthropomorphic walking Robot LOLA," IEEE/ASME Transactions on Mechatronics, vol. 14, no. 6, 2009.

[25] T. Buschmann, "Simulation and Control of Biped Walking Robots," $\mathrm{PhD}$ Thesis, Technical University of Munich, 2010.

[26] A. Liégeois, "Automatic Supervisory Control of the Configuration and Behavior of Multibody Mechanisms," IEEE Transactions on Systems, Man, and Cybernetics, vol. 7, no. 12, 1977.

[27] T. Buschmann, V. Favot, S. Lohmeier, M. Schwienbacher, and H. Ulbrich, "Experiments in Fast Biped Walking," in IEEE International Conference on Mechatronics, 2011.

[28] F. Sygulla, F. Ellensohn, A.-C. Hildebrandt, D. Wahrmann, and D. Rixen, "A Flexible and Low-Cost Tactile Sensor for Robotic Applications," in IEEE International Conference on Advanced Intelligent Mechatronics, 2017. 